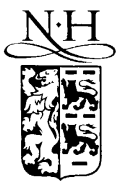

ELSEVIER

Surface Science 477 (2001) 25-34

\title{
Nanotribology of carbon based thin films: the influence of film structure and surface morphology
}

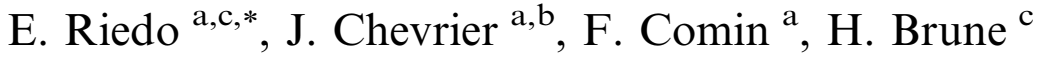 \\ ${ }^{a}$ ESRF, BP220, F-38043 Grenoble Cedex, France \\ ${ }^{\mathrm{b}}$ LEPES CNRS, F-38043 Grenoble, France \\ ${ }^{\mathrm{c}}$ EPFL, CH-1015 Lausanne, Switzerland
}

Received 6 August 2000; accepted for publication 22 December 2000

\begin{abstract}
The tribological behavior of carbon based thin films is strongly influenced by their chemical composition, polycrystalline structure and surface morphology. We present friction measurements on laser deposited amorphous carbon and carbon nitride $\left(\mathrm{CN}_{x}\right)$ thin films using atomic force microscopy. We studied the friction behavior of these films in relation with their structure and surface morphology resulting from the applied deposition parameters. We found high nanoscopic friction for amorphous carbon thin films, medium friction for $\mathrm{CN}_{x}$ and very low friction for graphite. Finally we discuss our findings in terms of the microscopic mechanisms of energy dissipation underlying the observed friction behavior. (c) 2001 Elsevier Science B.V. All rights reserved.
\end{abstract}

Keywords: Atomic force microscopy; Friction; Tribology; Carbon; Nitrides; Raman scattering spectroscopy; Surface structure, morphology, roughness, and topography

\section{Introduction}

The understanding of the mechanisms that control the friction behavior of different carbon compounds is an important goal, both from a fundamental physics perspective and for technological applications. Diamond-like carbon (DLC) thin films combine excellent technological properties, such as high hardness, good thermal conductivity, good wear resistance and chemical inertness [1]. Carbon nitride $\left(\mathrm{CN}_{x}\right)$ thin films prom-

\footnotetext{
${ }^{*}$ Corresponding author. Address: EPFL, CH-1015 Lausanne, Switzerland. Fax: +41-21-6933604.

E-mail address: elisa.riedo@epfl.ch (E. Riedo).
}

ise even more attractive properties like an improved hardness and elasticity and a lower friction coefficient $[2,3]$. Owing to their unique properties these films have found a wide range of applications as optical coatings, protective coatings for magnetic hard disks and biocompatible coatings $[4,5]$.

For all these technological applications DLC and $\mathrm{CN}_{x}$ thin films need excellent tribological and mechanical properties. In particular the magnetic recording industry has announced that for the next generation of hard disk drives very thin coatings with very low friction coefficient and extremely good wear resistance are required [3]. Although many efforts have been done to study macroscopic friction on these films, the basic understanding of 
friction and the relation of the tribological properties with the structure of the films is still on a poor level. To achieve this understanding it is necessary to study friction on the nanoscopic level. Such studies are expanding now because nanotechnology is beginning to build devices that are so miniaturized that their functionality relies on the microscopic friction properties of the materials. Moreover, the development of the atomic force microscope (AFM) has allowed the experimental investigation of friction localized on very small contact area.

This work provides a study of friction behavior at a nanoscopic scale for different amorphous carbon and $\mathrm{CN}_{x}$ thin films. In particular we show how the growth parameters and the resulting polycrystalline structure and surface morphology of the films influence the nanotribological behavior. Frictional forces between a silicon probe tip and the carbon compounds are found to be lower for the $\mathrm{CN}_{x}$ thin films than for the DLC films, however, highly oriented pyrolytic graphite (HOPG) has still lower frictional forces by one order of magnitude. Working in the normal load regime where interfacial wear is absent, we have studied the mechanisms of energy dissipation at the origin of the observed friction behavior in our samples.

\section{Experimental}

The HOPG surfaces used in this study were freshly cleaved in air right before the experiment. Amorphous carbon thin films were grown by pulsed laser deposition in an ultra high vacuum chamber at the base pressure of $p=10^{-9}$ Torr with laser fluences in the range $I_{\mathrm{L}}=1-12 \mathrm{~J} / \mathrm{cm}^{2}$, as described elsewhere [6,7]. In a previous study [6] we have characterized these films by X-ray photoelectron spectroscopy, electron energy loss spectroscopy and Raman spectroscopy revealing an increasing fraction of $\mathrm{sp}^{3}$ hybridization with increasing laser fluence $\left(34 \%\right.$ at $I_{\mathrm{L}}=1 \mathrm{~J} / \mathrm{cm}^{3}$ to $53 \%$ at $\left.I_{\mathrm{L}}=12 \mathrm{~J} / \mathrm{cm}^{2}\right)$. The $\mathrm{CN}_{x}$ films were grown in a nitrogen atmosphere of $100 \mathrm{~m}$ Torr yielding an atomic nitrogen content in the range $0.2<x<0.3$.
This composition corresponds to an increase in $\mathrm{N}-\mathrm{sp}^{3} \mathrm{C}$ bonded sites from $36 \%$ to $53 \%$ [7].

The degree of local order in the samples was investigated by means of micro-Raman spectroscopy. Micro-Raman measurements were performed using a LabRam Infinity Dilor apparatus probing an area of the order of a few $\mu \mathrm{m}^{2}$ and using light with a wavelength of $633 \mathrm{~nm}(\mathrm{He}-\mathrm{Ne}$ laser) or $458 \mathrm{~nm}$ (Ar laser), respectively.

Surface morphology studies and the first part of the friction measurements were performed with an AFM (Digital Instrument Nanoscope IIIa) operating in air and in contact mode. A beam deflection scheme with a four-quadrant photodetector allows simultaneous measurement of normal displacement and torsion of the cantilever, in this way topography and lateral forces are imaged simultaneously. We used silicon nitride cantilevers with a V-shape, narrow legs and a length of $100 \mu \mathrm{m}$. The silicon nitride tips have pyramidal geometry, radii of about $10 \mathrm{~nm}$ and a nominal normal force constant $k=0.38 \mathrm{~N} / \mathrm{m}$. We performed the first set of friction measurements on sliding distances of $50 \mathrm{~nm}$, with sliding speed of $0.4 \mu \mathrm{m} / \mathrm{s}$ and an applied normal load of $12 \mathrm{nN}$ as determined by force-distance curves. The second set of friction measurements, that is friction-load curves, were performed with an AFM from Park Scientific Instruments (model AutoProbe ${ }^{\mathrm{TM}}$ M5), also equipped with a four-quadrant photodetector. We used an ultralever $180 \mu \mathrm{m}$ long, with a nominal normal force constant of $0.40 \mathrm{~N} / \mathrm{m}$. This cantilever has a silicon conical tip, about $6 \mu \mathrm{m}$ high and with a radius of curvature of $\approx 10 \mathrm{~nm}$. The sliding speed was set to $2 \mu \mathrm{m} / \mathrm{s}$ and the applied loads ranged from 15 to $20 \mathrm{nN}$, well within the wearless friction regime $[8,9]$. The accuracy of the absolute values of the forces is limited by two factors. Firstly the large error in the spring constants (normal, lateral and torsional) of the cantilever. Secondly the presence of additional forces, difficult to estimate, between the tip and the sample surface, such as: van der Waals forces, attractive and repulsive electrostatic forces and capillary forces due to the thin water layer often present in an ambient environment [8]. However, relative changes in friction can be determined quite accurately by using the same cantilever and tip under identical envi- 
ronmental conditions during all the measurements. No evidence of tip and sample wear (seen as a change of friction with test duration on the same scanned area) was observed in the course of the experiments. All the experiments were carried out at room temperature and in air, with a relative humidity of $40 \%$.

\section{Results}

\subsection{Local order}

Fig. 1 shows typical Raman spectra of $\mathrm{CN}_{x}$ $(0.2<x<0.3)$ thin films and of two kinds of DLC thin films with high $(53 \%)$ and low $(34 \%)$ content of $\mathrm{sp}^{3}$ hybridization (in what follows we label these samples HDLC and LDLC, respectively). The Raman spectrum of the HDLC film exhibits a single and asymmetric broad peak. The LDLC film and the $\mathrm{CN}_{x}$ films show a second shoulder at lower wave numbers $\left(\sim 1360 \mathrm{~cm}^{-1}\right)$. This second peak increases in intensity in the DLC films with decreasing the laser fluence during deposition. The peak located at $1550 \mathrm{~cm}^{-1}$ (the so-called $\mathrm{G}$ peak, $\mathrm{G}$

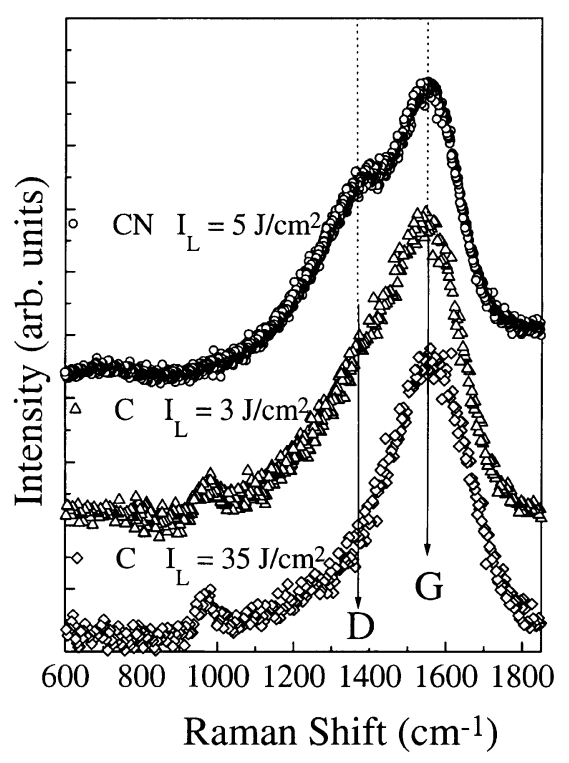

Fig. 1. Micro-Raman spectra of DLC films grown with laser fluences of 35 and $3 \mathrm{~J} / \mathrm{cm}^{2}$ and $\mathrm{CN}_{x}$ films grown with $5 \mathrm{~J} / \mathrm{cm}^{2}$ (from the bottom to the top). for graphite) corresponds to the symmetric $E_{2 g}$ vibrational mode in graphite-like materials. The $\mathrm{D}$ band (D for disorder) at about $1360 \mathrm{~cm}^{-1}$ arises from the limitations in the graphite domain size induced by grain boundaries or imperfections, such as substitutional $\mathrm{N}$ atoms, $\mathrm{sp}^{3}$ carbon, or other impurities [10]. The D band appears at a minimum size of the crystalline domains of 15-20 $\AA$ [11], for larger grain sizes it is sensitive to the local disorder in the material. For smaller grain sizes the $\mathrm{D}$ band changes its form and decreases in intensity. Analyzing the spectra in Fig. 1 it results that the HDLC sample presents a markedly amorphous nature with a mixture of $\mathrm{sp}^{2}$ and $\mathrm{sp}^{3}$ hybridization [12]. In Fig. 1 we can observe from the bottom to the top spectrum, the evolution from an amorphous towards a more ordered structure, respectively in the samples HDLC, LDLC and $\mathrm{CN}_{x}$. In fact, the raising of a D band means that the size of crystalline domains is becoming larger than $20 \AA[11,12]$. The peak at about 980 $\mathrm{cm}^{-1}$ arises from the $\mathrm{SiC}$ layer that is formed at the interface $\mathrm{Si} / \mathrm{C}$. The Raman spectra of the $\mathrm{CN}_{x}$ films in the range $2000-2400 \mathrm{~cm}^{-1}$ show a small peak at about $2220 \mathrm{~cm}^{-1}$ corresponding to the $\mathrm{C} \equiv \mathrm{N}$ stretching mode [13].

\subsection{Topography}

In Fig. 2 we show an AFM image revealing the morphology of a $1 \times 1 \mu^{2}$ area of a DLC sample.

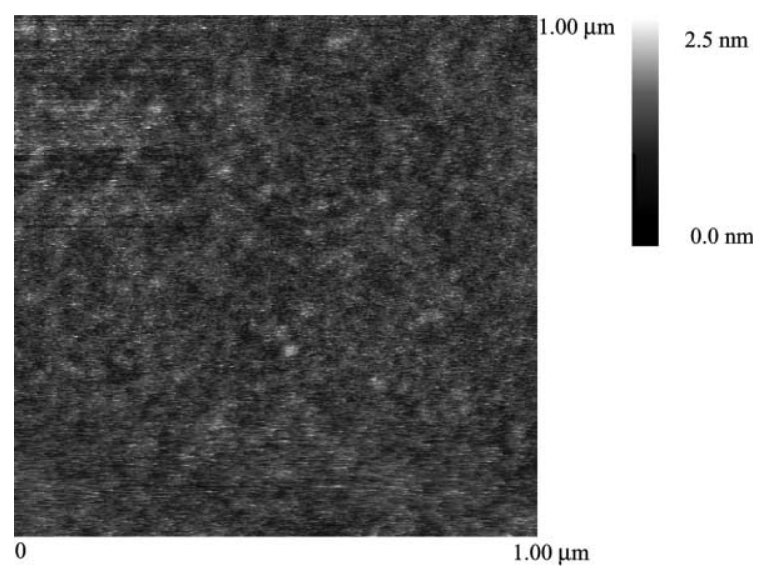

Fig. 2. Typical AFM topography image of DLC films. 
Based on such images one readily calculates the root mean square ( $\mathrm{rms})$ roughness $R_{q}$ defined as

$R_{q}=\left(\sum\left(z_{\mathrm{i}}-z_{\text {average }}\right)^{2} / N\right)^{1 / 2}$,

where $z_{\text {average }}$ is the average of the $z$ height values within a given area, $z_{\mathrm{i}}$ is the individual $z$ value and $N$ is the number of pixels within the given area. The resulting rms roughness is $2.0 \pm 0.5 \AA$ for the HDLC samples and $5.0 \pm 0.5 \AA$ for the LDLC samples. The rms roughness of the silicon substrate is $2.0 \pm 0.5 \AA$, indicating perfect replication of the substrate morphology by the films.

The surface morphology of the $\mathrm{CN}_{x}$ films is shown for $x=0.23$ in Fig. 3(a) and (b) in two magnifications. These images as well as the images obtained from other $\mathrm{CN}_{x}$ films $(0.2<x<0.3)$, show that the surface is covered homogeneously by clusters of about $50 \mathrm{~nm}$ in diameter. Formation of nanoparticles produced from a supersaturated vapor by laser ablation is well known in the literature $[13,14]$. The relatively high pressure of nitrogen gas confines the plasma produced by laser
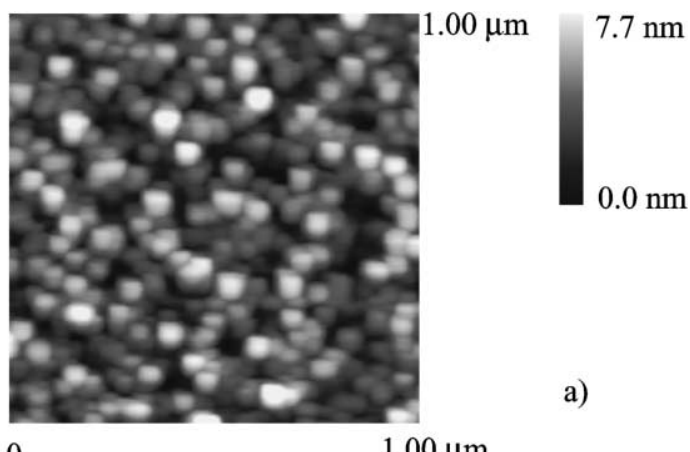

0

$1.00 \mu \mathrm{m}$

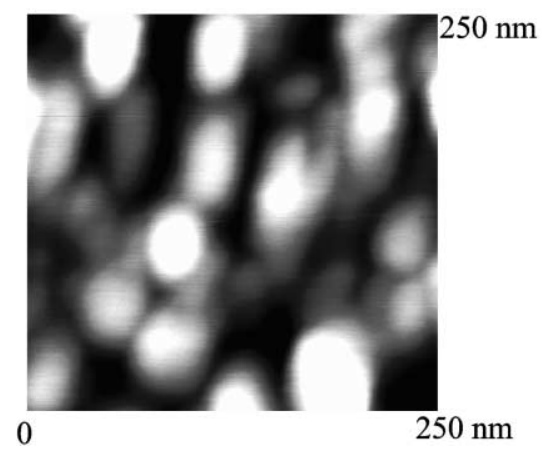

a)

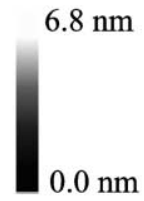

b)

Fig. 3. AFM topography images of $\mathrm{CN}_{x}$ films with $x=0.23$. ablation of graphite and it condenses to form clusters. The rms roughness of the $\mathrm{CN}_{x}$ samples is between 10 and $16 \AA$, slightly greater than in the DLC samples.

In order to obtain more quantitative information about the morphology of our carbon based samples we have calculated from the AFM images the height-height correlation function $H(r)$, defined as $H(r) \equiv\left[\left\langle\left(z(\boldsymbol{r})-z\left(\boldsymbol{r}^{\prime}\right)\right)^{2}\right\rangle_{\text {area }}\right]$, where $z(\boldsymbol{r})$ and $z\left(\boldsymbol{r}^{\prime}\right)$ are the heights at the coordinates $\boldsymbol{r}=$ $(x, y)$ and $\boldsymbol{r}^{\prime}=\left(x^{\prime}, y^{\prime}\right)$ and $r=\left|\boldsymbol{r}-\boldsymbol{r}^{\prime}\right|$ [14]. The brackets in this definition indicate a spatial average. Because the surface morphology of our samples is isotropic, it suffices to calculate the one-dimensional instead of the two-dimensional height-height correlation function. As the AFM fast scan direction $x$ is much less affected from $1 / f$ noise than the slow direction $y$, we estimated $H(r)$ from $N_{x} \times N_{y}$ pixel topographies after averaging $\left(z(\mathbf{r})-z\left(\mathbf{r}^{\prime}\right)\right)^{2}$ on single rows along $x$ direction and then averaging the $N_{y}$ values so obtained [16],

$$
\begin{aligned}
H(r) & =H(m) \\
& =\frac{1}{N_{y}\left(N_{x}-m\right)} \sum_{j=1}^{N_{y}} \sum_{i=1}^{N_{x}-m}[z(i+m, j)-z(i, j)]^{2},
\end{aligned}
$$

where $m$ is the distance between the two points in $x$-direction in pixel units.

In Fig. 4 we show a typical $H(r)$ curve resulting from DLC samples, while in Fig. 5(a) and (b) we

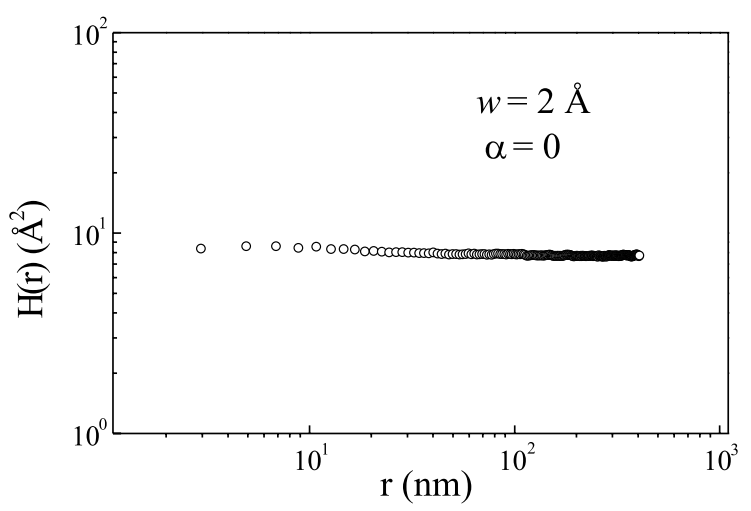

Fig. 4. Typical height-height correlation function $H(r)$ for DLC surfaces. 


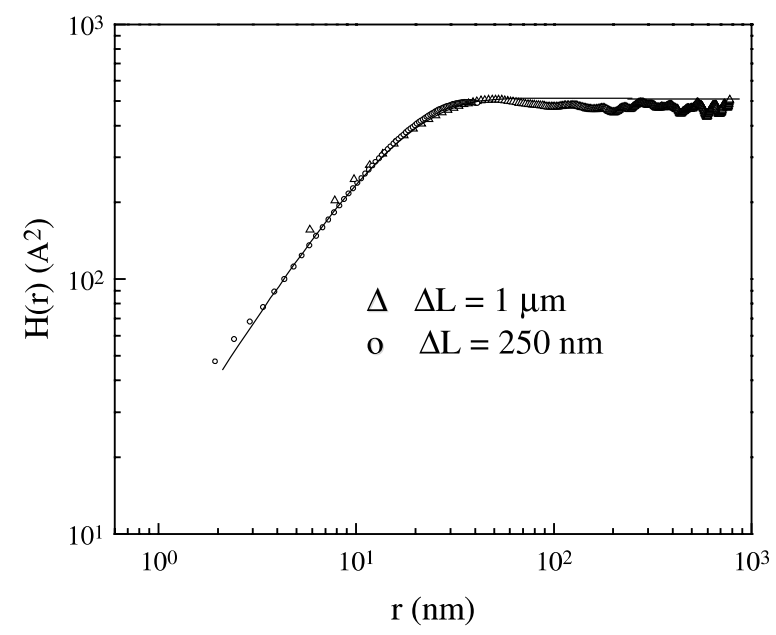

a)

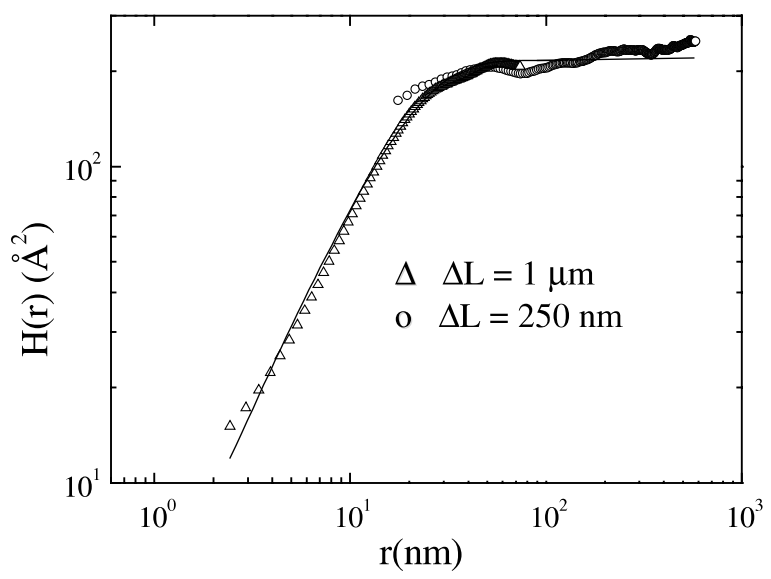

b)

Fig. 5. Typical height-height correlation functions $H(r)$ for (a) $\mathrm{CN}_{x}$ films with $x=0.23$ and (b) $\mathrm{CN}_{x}$ films with $x=0.28$.

report the results obtained from the samples $\mathrm{CN}_{0.23}$ and $\mathrm{CN}_{0.28}$. We fitted the results with the function $G(r)$ used to describe self-affine structures $[15,16]$

$G(r)=2 w^{2}\left[1-\exp \left(-(r / \xi)^{2 \alpha}\right)\right]$,

where $w(r, t)$ is called interface width and corresponds to the rms roughness $R_{q}$ defined in Eq. (1) at a given time $t$ and for a straight line $r$ on the surface, $\alpha$ is the roughness exponent and $\xi$ is the lateral correlation length. For the DLC samples we obtained $\alpha=0$ [15], in agreement with the smoothness of the surface reported in Fig. 1 and with the constant value of $H(r)$. The growth of the $\mathrm{CN}_{x}$ thin films differs from the growth of DLC films in so far as we now deal with deposition of clusters and no more of atoms [17]. The resulting correlation lengths $\xi$ for the $\mathrm{CN}_{x}$ films $(\sim 150-190$ $\AA$ ) are of the same order of magnitude as the cluster diameter. This means that single clusters show the scaling behavior typical of self-affine surfaces, while for scaling length larger than the cluster diameter, the heights are uncorrelated and the surface appears as smooth as the one of the DLC films. Other authors have found that carbon films deposited under deposition conditions similar to ours possess a fractal structure $[18,19]$.

\subsection{Friction}

We have studied nanoscale frictional forces between an AFM tip and the surface of the following 
carbon compounds: HOPG, DLC with different percentages of $\mathrm{sp}^{3}$ hybridization $(34-53 \%)$ and $\mathrm{CN}_{x}$ with different content of nitrogen $(0.2<$ $x<0.3$ ). In Fig. 6 we show lateral force images on $1 \times 1 \mu \mathrm{m}^{2}$ of the surface of a $\mathrm{CN}_{x}$ film taken simultaneously with the topographical image.

In addition to these images giving the spatial variation of friction force $\left(F_{\mathrm{F}}\right)$, we have taken

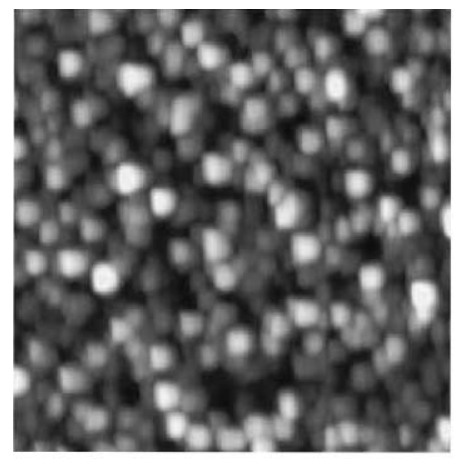

\section{Topography \\ Scan size: $1 \mu \mathrm{m}$ \\ $\mathrm{Z}$ range $7.707 \mathrm{~nm}$}

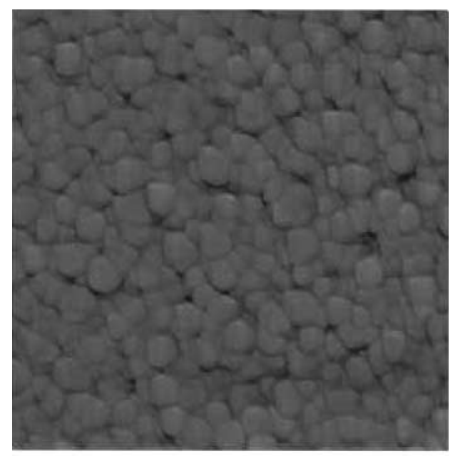

Friction $(\rightarrow)$

Scan size: $1 \mu \mathrm{m}$

$\mathrm{Z}$ range $0.8 \mathrm{~V}$

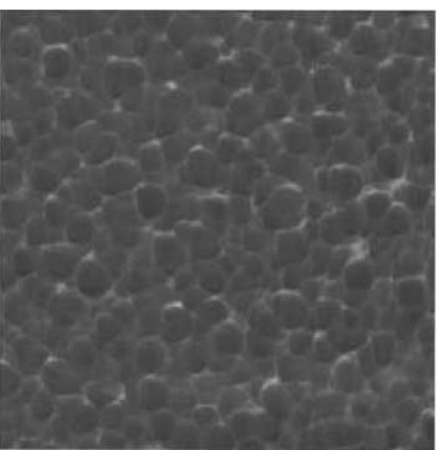

Friction $(<-)$

Scan size: $1 \mu \mathrm{m}$

$\mathrm{Z}$ range $1 \mathrm{~V}$ single lateral force scans to investigate the relative values of $F_{\mathrm{F}}$ for different samples as a function of applied load. For sake of comparison these measurements were taken out with the same tip and under the same environmental conditions.

Fig. 7(a)-(c) shows the friction forces for HOPG, $\mathrm{CN}_{x}$ and DLC samples, respectively, applying a

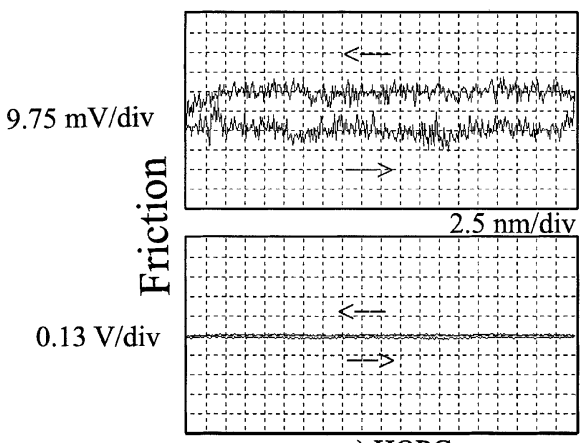

a) HOPG

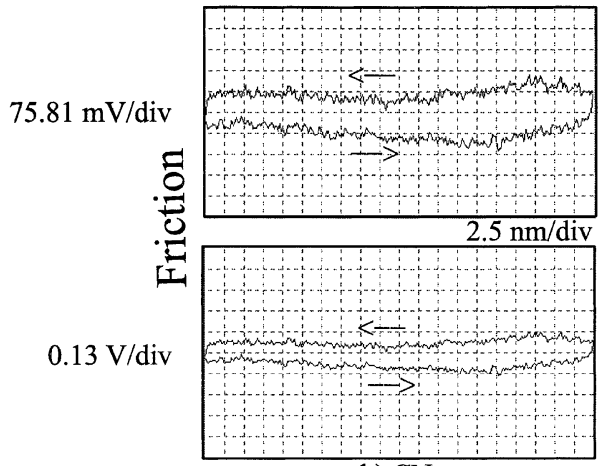

b) $\mathrm{CN}_{\mathrm{x}}$

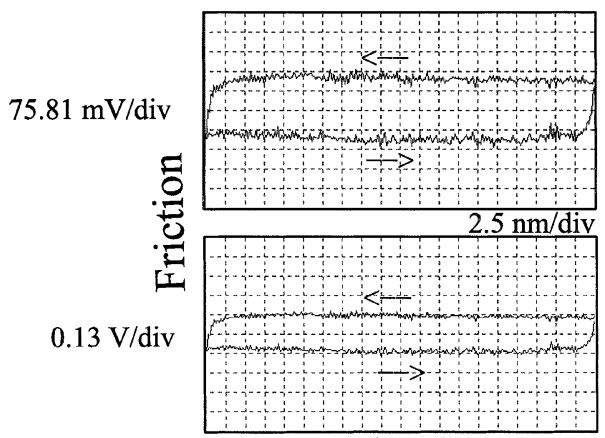

c) DLC

Fig. 7. Friction forces for the samples of (a) graphite (HOPG), (b) $\mathrm{CN}_{x}$ and (c) DLC. Each graph presents a scan width of 50 $\mathrm{nm}$ and two force signals corresponding to foreword and backward scan.
Fig. 6. Topographical image (top) of a $\mathrm{CN}_{x}$ film taken simultaneously with friction image in the forward $(\rightarrow)$ and backward $(\leftarrow)$ scan direction (middle and bottom image respectively). 
load of $12 \mathrm{nN}$. For each surface we show the forward and backward scan with the friction force being proportional to the difference between the two. The upper graphs have an enlarged friction scale to show the generally smooth fine structure in the lateral forces, revealing that the usual stick and slip behavior that we observed on mica (not shown here), is not observed, at the probed length scales, on our samples. The lower graphs shows the friction force on an absolute scale indicating that $F_{\mathrm{F}}$ $\left(\right.$ DLC) $>F_{\mathrm{F}}\left(\mathrm{CN}_{x}\right)>F_{\mathrm{F}}(\mathrm{HOPG})$. Furthermore, we have observed that friction forces are almost identical for the different DLC films, independent of the percentage of $\mathrm{sp}^{3}$ hybridization (from $34 \%$ to $53 \%$ ). The friction is the same also in all the investigated $\mathrm{CN}_{x}$ films.

To enable more accurate quantitative comparison of $F_{\mathrm{F}}$ between the samples, we have measured the friction forces as a function of applied loads. Fig. 8 displays the friction-load plots for the measurements made on graphite, $\mathrm{CN}_{x}$ and DLC surfaces. Each data point represents an average of the friction force $\left(F_{\mathrm{F}}\right)$ measured on $1 \mu \mathrm{m}$ of the surface at a fixed applied load $\left(F_{\mathrm{L}}\right)$, for increasing and decreasing loads. The data shown here for decreasing loads extend to negative applied loads due to adhesion between the tip and the sample [9].

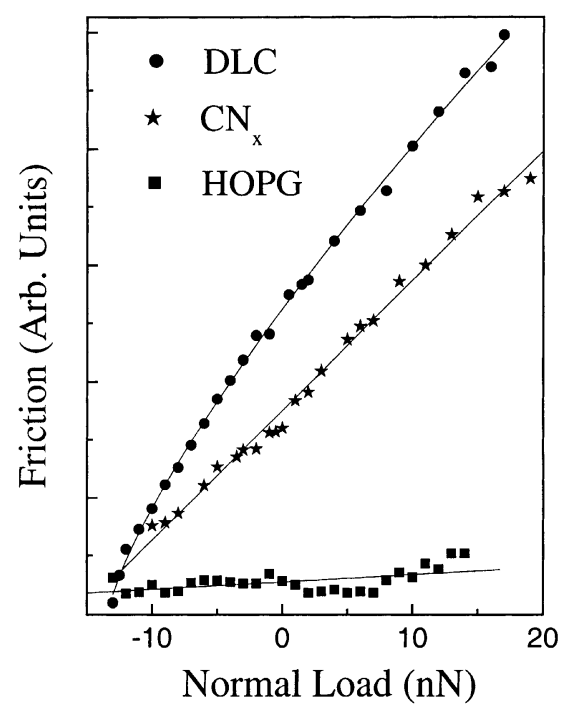

Fig. 8. Plots of friction force versus applied load for graphite $(\boldsymbol{\square}), \mathrm{CN}_{x}(\star)$ and amorphous carbon $(\bullet)$ surfaces.
Classically, the slope of the friction-load curve determines the friction coefficient $\mu$, as described by Amontons' law: $F_{\mathrm{F}}=\mu F_{\mathrm{L}}$. However, in the case of a single-asperity contact, Amontons' law is no longer valid and the friction-load curves, for low loads, show a non-linear behavior. Some authors [9] have found a $F_{\mathrm{F}} \sim F_{\mathrm{L}}^{2 / 3}$ dependence of the single asperity friction on the load for some carbon samples. Furthermore in presence of attractive forces, the same authors showed that, for not too soft materials, $F_{\mathrm{L}}$ has to be replaced, as a first approximation, by an effective force $F_{\mathrm{n}}=F_{\mathrm{L}}+F_{0}$, where $F_{0}$ is the adhesive force which can in principle be determined by force-distance curves. As shown in Fig. 8, we found a linear behavior for graphite and $\mathrm{CN}_{x}$ films, whereas the DLC films present an excellent agreement with the $F_{\mathrm{F}} \sim F_{\mathrm{n}}^{2 / 3}$ law [9]. Since we used the same tip in all the measurements, and we did not observe interfacial wear, these different force laws are probably due to the different surface morphology of our samples. The DLC films, in fact, present a rather smooth surface in comparison with the $\mathrm{CN}_{x}$ films and the HOPG surface and for this reason a single contact regime with the tip is more likely.

The friction coefficient was determined by a linear regression of $F_{\mathrm{F}}$ versus $F_{\mathrm{L}}$; data showing a curvature at low loads were excluded in the evaluation of the slope. We have derived the following results for the friction coefficient: $\mu=(0.009 \pm$ $0.003)$ a.u. for HOPG, $\mu=(0.167 \pm 0.003)$ a.u. for the $\mathrm{CN}_{x}$ films and $\mu=(0.208 \pm 0.003)$ a.u. for the DLC films, where the error represents the standard deviation and a.u. are absolute arbitrary units, but permitting relative comparison. We do not give absolute values for the friction coefficients because of the large uncertainty in the calibration of the lateral forces.

\section{Discussion}

Recent reviews [21,22] of the tribological properties of DLC films and their modified forms show that, in all environments, the macro-tribological behavior of DLC is controlled by an interfacial transfer layer formed during friction. This layer is formed by a friction-induced modification of the 
top layer of the DLC film into a material of low shear strength. In spite of this comprehension at a macroscopic scale, very little is known on the mechanisms that control friction at a nanoscopic level. Furthermore, it has been demonstrated [23] that for graphite and hydrogenated carbon films the macro-tribological behavior is not directly comparable with the nanotribological one, due to dependence of wear, plastic deformation and delamination on the contact area. Some authors [9] have reported that nanofriction (against tungsten and $\mathrm{SiN}$ AFM tips) increases with going from graphite via diamond to amorphous carbon and finally to $\mathrm{C}_{60}$.

We performed our experiment in air since several authors have demonstrated for different carbon compounds, comparable friction results in air (humidity $<75 \%$ ) and in dry argon atmosphere $[9,24,25]$. To avoid plastic deformation and wear we restricted us to normal loads $F_{\mathrm{L}}<20 \mathrm{nN}$. In this load regime we did not observe any evidence of interfacial wear, which would have led to an increase in pull-off forces as a consequence of the increase of the tip radius or the formation of readily detectable wear defects on the surface.

Friction can be described as the dissipation of energy at a sliding interface. To study the mechanisms responsible for a particular frictional behavior we have thus to discuss the possible pathways of energy dissipation. Since we performed our experiments in air, adhesion could in principle play a role in the friction behavior of these films. However, from pull-off force measurements we found that the adhesion force $F_{0}$ has the opposite trend of the friction coefficient in our samples, that is, $F_{0}$ (DLC) $<F_{0}\left(\mathrm{CN}_{x}\right)<F_{0}$ (HOPG). This order of the pull-off forces is confirmed by the applied loads where $F_{\mathrm{F}}$ is zero (see crossing of $x$-axis in Fig. 8). These results indicate that adhesion is not directly related to friction in the investigated carbon based materials.

In the wearless regime two additional fundamental mechanisms responsible for frictional properties have been considered in the literature. First, dissipation of friction energy can take place by emission of phonons [26-28]. The mechanical energy necessary to slide one surface against the other is dissipated into heat through excitation of lattice vibrations. The second fundamental contribution to frictional energy dissipation is electron-hole pair excitation [29]. Up to date it is not clear which mechanism dominates and only few theoretical and experimental studies [30-33] have addressed this topic. This is partially due to the fact that most experiments measure only macroscopic friction forces. Some authors have proposed that the electronic mechanism may be an important mechanism for energy dissipation [20,29]. However, in insulating systems the phononic contribution can be usually considered as dominant.

In our experiment we investigated friction on the HOPG, on DLC and on $\mathrm{CN}_{x}$ films. Firstly, from the electron energy loss spectra of our samples $[6,7]$ we expect the same electronic behavior from $\mathrm{CN}_{x}$ films and DLC films rich of $\mathrm{sp}^{3}$ hybridization, instead a different behavior is expected from DLC films poor of $\mathrm{sp}^{3}$ hybridization. Secondly the DLC and $\mathrm{CN}_{x}$ films are insulating materials, while graphite has a quasi-metallic character. If the electronic mechanism were the dominant contribution to energy dissipation in the studied carbon compounds we would expect lower friction forces in the insulating films (DLC and $\mathrm{CN}_{x}$ films) compared to the quasi-metallic HOPG sample. Our results demonstrate that friction forces behave exactly opposite to this expectation and they are independent on the degree of $\mathrm{sp}^{3}$ hybridization in DLC films, thus we suggest that the friction behavior of the investigated carbon compounds is dominated by phononic mechanisms.

The very low nanofriction forces found in the HOPG sample can be explained with the low shear strength of the graphite layers. The different behavior of DLC and $\mathrm{CN}_{x}$ films could be due to their different morphological structure (see Figs. 2 and 3). Fig. 9 shows the line scans of friction and topography taken simultaneously on a $\mathrm{CN}_{x}$ film. From this figure it is clear that the $2 \mathrm{~nm}$ high features, appearing with a period of $16 \mathrm{~nm}$ in the topography, do not influence the friction behavior, which thus, seems to be uncorrelated to the film roughness. Therefore a more attractive explanation of the higher friction coefficient in the DLC films with respect to the $\mathrm{CN}_{x}$ films is that the 


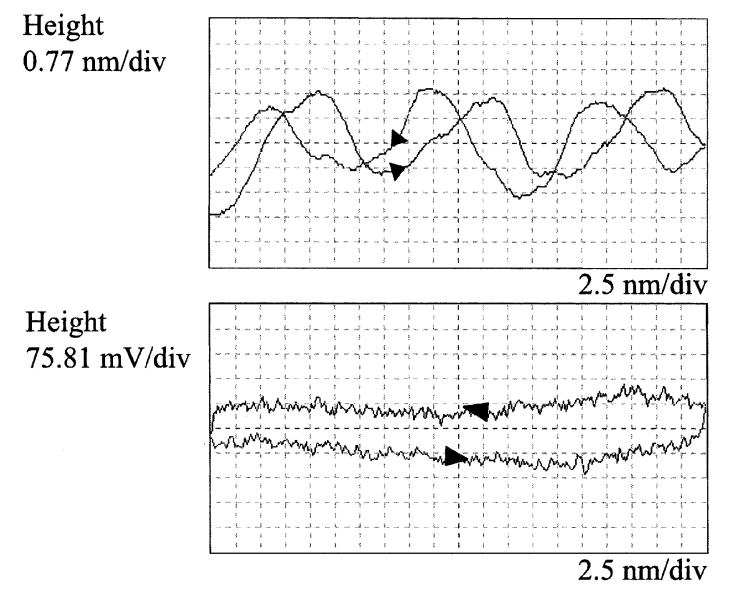

Fig. 9. Simultaneous profiles of friction and topography on a $50 \mathrm{~nm}$ scan width for a $\mathrm{CN}_{0.23}$ film.

presence of $\mathrm{N}$ atoms changes the polycrystalline structure of the carbon films (see Raman spectra in Fig. 1) and thus the number of phonon modes available for excitation. Other authors [3,34-37] have already pointed out that $\mathrm{CN}_{x}$ films are very hard and have better elastic and tribological properties than DLC films. Experimental and theoretical studies $[34,36]$ have demonstrated that these improved properties of the $\mathrm{CN}_{x}$ films are due to a "fullerene-like" microstructure with curved and intersecting basal planes. To decide whether this "fullerene-like" microstructure is responsible of a lower friction coefficient at a nanoscopic scale, molecular dynamics calculations could be very helpful.

It was reported [38] that friction and wear of diamond films are reduced on a macroscopic scale by adding $\mathrm{sp}^{2}$ carbon bonds. This observation is not confirmed by us on the nanoscale where the friction behavior on DLC films was seen to be independent of the degree of $\mathrm{sp}^{3}$ hybridization.

\section{Conclusions}

We studied the tribological properties of different carbon based thin films which are very promising materials in hard coating technology. We could investigate the nanoscale friction behavior as a function of film chemical composition, polycrystalline structure and morphology, putting thus constrains on the number of dissipative processes acting in absence of wear. We found high microscopic friction for amorphous carbon thin films, medium friction for carbon nitride and very low friction for graphite. We have derived that these observed differences are neither related to the adhesion, nor to surface morphology, instead we suggest that different phonon excitation in the amorphous carbon and $\mathrm{CN}_{x}$ thin films is at the origin of the found friction behavior.

\section{Acknowledgements}

The authors would like to thank the Progetto Ricerca Avanzata CLASS of the INFM. We wish to thank S. Decossas for his help with the AFM. Also the use of the micro-Raman spectroscopy facilities with the help of A.M. Bonnot is gratefully acknowledged.

\section{References}

[1] S. Neuville, A. Matthews, MRS Bulletin, 22, September 1997.

[2] T.W. Scharf, R.D. Ott, D. Yang, J.A. Barnard, J. Appl. Phys. 85 (1999) 3142.

[3] B. Wei, B. Zhang, K.E. Johnson, J. Appl. Phys. 83 (1998) 2491.

[4] J. Robertson, Prog. Solid State Chem. 21 (1991) 199.

[5] A. Grill, Diam. Relat. Mater. 8 (1999) 428.

[6] E. Riedo, F. Comin, J. Chevrier, F. Schmitüsen, S. Decossas, M. Sancrotti, Surf. Coat. Technol. 125 (2000) 124.

[7] E. Riedo, F. Comin, J. Chevrier, A.M. Bonnot, J. Appl. Phys. 88 (2000) 4365.

[8] U.D. Schwartz, H. Bluhm, H. Hölsher, W. Allers, R. Wiesendanger, in: B.N.J. Persson, E. Tosatti (Eds.), The Physics of Sliding Surfaces, Kluwer Academic Publishers, Dordrecht, 1996.

[9] U.D. Schwartz, O. Zwörner, P. Koster, R. Wiesendanger, Phys. Rev. B 56 (1997) 6987.

[10] J.K. Kaufman, S. Metin, D.D. Saperstein, Phys. Rev. B 39 (1989) 13053.

[11] A. Kumar, R.B. Inturi, MRS Symp. Proc. 397 (1996) 289.

[12] J. Robertson, Prog. Solid State Chem. 21 (1991) 199.

[13] W.A. deHeer, Rev. Mod. Phys. 65 (1993) 611.

[14] T. Sasaki, X. Zeng, N. Koshizaki, MRS Symp. Proc. 526 (1998) 67. 
[15] A.L. Barabasi, H.E. Stanley, Fractal Concepts in Surface Growth, Cambridge University Press, Cambridge, 1995.

[16] H.N. Yang, Y.P. Zhao, A. Chan, T.M. Lu, G.C. Wang, Phys. Rev. B 56 (1997) 4224.

[17] G.S. Bales, R. Bruinsama, E.A. Eklund, R.P.U. Karunasiri, J. Rudnuck, A. Zangwill, Science 249 (1990) 264.

[18] I.V. Zolotukhin, Yu.V. Sokolov, V.P. Ievlev, Phys. Solid State 40 (1998) 539.

[19] R. Buzio, E. Gnecco, C. Boragno, U. Valbusa, P. Piseri, E. Barborini, P. Milani, Surf. Sci. Lett. 444 (2000) L1.

[20] P.B. Merrill, S.S. Perry, Surf. Sci. 418 (1998) 342.

[21] A. Grill, Surf. Coat. Technol. 94/95 (1997) 507.

[22] M.N. Gardos, Synthetic Diamond: Emerging CVD Science and Technology, Wiley, NY, 1993, p. 143.

[23] E. Liu, B. Blanpain, J.-P. Celis, J.R. Roos, J. Appl. Phys. 84 (1998) 4859.

[24] C.M. Mate, Wear 168 (1993) 17.

[25] M. Bingelli, C.M. Mate, Appl. Phys. Lett. 65 (1994) 415.

[26] M. Cieplak, E.D. Smith, M.O. Robbins, Science 265 (1994) 1209.
[27] M.S. Tomassone, J.B. Sokoloff, A. Widom, J. Krim, Phys. Rev. Lett. 79 (1997) 4798.

[28] J. Krim, Langmuir 12 (1996) 4564.

[29] B.N.J. Persson, Phys. Rev. B 44 (1991) 3277.

[30] R.J.A. van den Oetelaar, C.F.J. Flipse, Surf. Sci. 384 (1997) L828.

[31] M. Enachescu, R.J.A. van den Oetelaar, R.W. Carpick, D.F. Ogletree, C.F.J. Flipse, M. Salmeron, Phys. Rev. Lett. 81 (1998) 1887.

[32] C. Daly, J. Krim, Phys. Rev. Lett. 76 (1996) 803.

[33] M.D. Perry, J.A. Harrison, J. Phys. Chem. B 101 (1997) 1364.

[34] H. Sjostrom, S. Stafstrom, M. Bonan, J.E. Sundgren, Phys. Rev. Lett. 75 (1995) 1336.

[35] T.W. Scharf, H. Deng, J.A. Barnard, J. Appl. Phys. 81 (1997) 5393.

[36] N. Hellgren, M.P. Johansson, E. Broitman, L. Hultman, J.E. Sundgren, Phys. Rev. B 59 (1999) 5162.

[37] G.A. Amaratunga, M. Chhowalla, C.J. Kiely, I. Alexandrou, R. Aharonov, R.M. Devenish, Nature 383 (1996) 321.

[38] T. Le Huu, M. Schmitt, D. Paulmier, Surf. Sci. 433-435 (1999) 690. 